\title{
EVALUATION OF POSSIBLE GALACTAGOGUIC ACTIVITY OF A SELECTED GROUP OF SRI LANEAN MEDICINAL PLANTS
}

\author{
KAMANI H. TENNEKOON", S. JEEVATHAYAPARAN ${ }^{*}$ AND ERIC H. \\ KARUNANAYAKE ${ }^{* *}$ \\ Department of Physiology and Department of Biochemistry , Faculty of Medicine, \\ University of Colombo, Colombo.
}

\author{
(Date of receipt : $\quad 06$ August 1991) \\ (Date of acceptance : 25 March 1992)
}

\begin{abstract}
In view of the potential benefits of herbal medicinal plants in improving lactation, a selected group of Sri Lankan plants used in the traditional system of Medicine as galactagogues were evaluated for possible galactagoguic activity in Sprague Dawley rats. Extracts of Nigella sativa, Dregea volubilis, Ipomea digitata, Borassus flabellifer, Corriandrum sativum, Momordica charantia, Carica papaya, Centella usiatica and sillium sativum were administered orally to lactating rats for 1 week from the 5 th day after delivery. The number of littermates were limited to 7 per mother. Weight gain and milk intake in the litter on the 7th day of the experiment were compared with control groups that received equivalent amounts of vehicle under identical conditions. Significant galactagoguic activity was not detected in any of the plant extracts studied, although the doses of extracts used were adequate to concentrate possible active principles. However, a seasonal variation of the active principle or possible inactivation of the galatagoguic activity in the digestive system cannot be excluded.
\end{abstract}

\section{Introduction}

Herbal medicinal plants are recommended by practitioners of the traditional systems of medicine for increasing milk secretion in lactating women in several parts of the world. However, scientific evaluation of the plants for possible galactagoguic activity has been very limited. ${ }^{1}$ A cold water extract of Abroma augusta, ${ }^{2}$ crude alcoholic extract of Asparagus racemosus, ${ }^{3,4}$ crude powder of Leptadenia reticulata ${ }^{5}$ and seeds of Nigella sativa ${ }^{6,7}$ have been reported to possess galactagoguic activity. Weight gain of litter after administering the plant extract to lactating rats, changes in mammary histology or weight after administering the plant extract to oestrogen primed or post partum rats and milk yield in livestock after administering the plant in their diet were different indices of galactagoguic activity used in experiments. However, Tennekoon and Coworkers ${ }^{8}$ failed to observe a sigrificant galactagoguic activity in Asparagus falcatus administered to lactating Sprague Dawley rats. The present study was designed to investigate the possible galactagoguic activity of a selected group of Sri Lankan medicinal plants using lactating rats as a model.

Lactating Sprague Dawley rats were used as the animal model to evaluate possible galactagoguic activity of several plants which are recommended as galactagogues in 
traditional systems of medicine. Plants were selected from a worldwide literature search carried out prior to commencement of the project.

\section{Materials and Methods}

\section{Animal Experiments}

Three month old virgin female rats were paired with males. When they littered, number of littermates were reduced to 7 per mother. Mothers with their litter were randomly allocated into treatment $(n=6$ to 10$)$ and control $(n=6$ to 10$)$ groups. The day of littering was designated as D1 and the plant extract to the mothers in the treatment groups and the vehicle to the mothers in the control groups were administered via oral animal feeding tubes under transient light ether anaesthesia daily from D5 to D12. Weights of mothers and their littermates were recorded daily from D5 to D13 and weight gain per littermate from D5 to D13 as well as the percentage weight gain of littermates from D5 to D13 were compared between treatment groups and their respective control groups using Student's test. Further, milk intake per littermate was also compared between treatment and their respective control groups. On D12 after administering plant extract/vehicle to the mothers they were separated from their litter for 4 hours. At the end of 4 hours litter was weighted and mothers were given an intramuscular injection of syntocinon $(0.5 \mu / 100 \mathrm{~g}$ body wt.) and the litter was then allowed to suck. Litter was weighed again at the end of the suckling period and the milk intake per littermate computed using the difference of litterweight before and after suckling as the total railk intake.

The animal model selected was evaluated for suitability in investigating galactagoguic activity using a known galactagogue chlopromazine. Two experiments were conducted using chlopromazine $0.05 \mathrm{mg} / 100 \mathrm{~g}$ body wt. per day and twice a day. Chlopromazine was administered intramuscularly from D5 to D12 to mothers in the treatment groups while control mother received distilled water. Rest of the experimental procedure was as described above.

\section{Preparation of plant extracts and dosages}

Nigella sativa Linn. (Family: Ranunculaceae, S: Kaluduru, T: Simaishembu or Karunj cheeraham):

Concentrated aqueous extract: $250 \mathrm{~g}$ of dried seeds of $N$. sativa were boiled with $1000 \mathrm{ml}$ of water for $90 \mathrm{~min}$ and filtered. The filtrate $(320 \mathrm{ml})$ was administered at a dose of $1 \mathrm{ml} / 100 \mathrm{~g}$ body $\mathrm{wt}$. and the respective control group received an equivalent amount of distilled water.

Diluted aqueous extract: An aliquot of the concentrated aqueous extract was diluted with an equal volume of distilled water and administered at a dose of $1 \mathrm{ml} / 100 \mathrm{~g}$ 
body wt. and the respective control group received an equivalent amount of distilled water.

Ether extract: The residue of $N$. sativa seeds after obtaining the aqueous extract was extracted with cold ether. The extract was concentrated in vacuo and the concentrate was air dried and subsequently suspended in 5\% Tween in $0.9 \%$ saline. This was administered at a dose of $100 \mathrm{mg} / 100 \mathrm{~g}$ body weight and the respective control group received an equivalent amount of $5 \%$ Tween in $0.9 \%$ saline.

Dregea volubilis Linn. (Family: Asclepiadaceae, S: Anguna, T: Kurinja):

Concentrated aqueous extract: Mature leaves of $D$. volubilis (258 $\mathrm{g}$ ) were boiled with water $(1500 \mathrm{ml})$ for $90 \mathrm{~min}$ and then squeezed through muslin cloth and filtered. The filtrate $(690 \mathrm{ml})$ was administered at a dose of $1 \mathrm{ml} / 100 \mathrm{~g}$ body wt. and the respective control group received an equivalent amount of distilled water.

Diluted aqueous extract: An aliquot of the concentrated aqueous extract was diluted with an equal volume of distilled water and administered at a dose of $1 \mathrm{ml} / 100$ $\mathrm{g}$ body wt. and the respective control group received an equivalent amount of distilled water.

Ipomea digitata Linn. (Family: Convolvulaceae, S: Kiribadu, T: Nilappuchani):

Aqueous extract: Tuberous roots of $I$. digitata $(742 \mathrm{~g})$ were boiled with water (2000 $\mathrm{ml}$ ) for $90 \mathrm{~min}$ and then squeezed through muslin cloth and filtered. The filtrate was freeze dried and resuspended in distilled water to yield a solution of $50 \mathrm{mg} / \mathrm{m}$. This was administered at a dose of $1 \mathrm{ml} / 100 \mathrm{~g}$ body wt. and the respective control group received an equivalent amount of distilled water.

Borassus flabellifer Linn. (Family: Palmae, S: Thal, T: Panammaram):

Kernel of $B$. flabellifer was freeze dried and suspended in water to yield a solution of $40 \mathrm{mg} / \mathrm{ml}$ and this was administered at a dose of $1 \mathrm{ml} / 100 \mathrm{~g}$ body $\mathrm{rt}$. and the respective control group received an equivalent amount of distilled water.

Corriandrum sativum Linn. (Family: Umbelliferae, S: Kottamalli, T: Kotamalli):

$250 \mathrm{~g}$ of dried seeds of $C$. sativum were boiled with $1000 \mathrm{ml}$ of water for 2 hours and filtered. Filtrate $(250 \mathrm{ml})$ was administered at a dose of $1 \mathrm{ml} / 100 \mathrm{~g}$ body wt. and the respective control group received an equivalent amount of distilled water.

Momordica charantia Linn: (Family: Cucurbitaceae, S: Karawila, T: Pavakkayi):

Mature green fruits of $M$. charantia were liquidized after removing seeds and placentae $(500 \mathrm{~g})$ and then squeezed though muslin cloth and filtered. The filtrate 
$(250 \mathrm{ml})$ was administered at a dose of $1 \mathrm{ml} / 100 \mathrm{~g}$ body wt. The respective control group received an equivalent amount of distilled water.

Carica papaya Linn. (Family: Caricaceae, S: Papol, T: Pappali):

Mature unripe fruits of C. papaya $(500 \mathrm{~g})$ were liquidized and then squeezed through muslin cloth and filtered. The filtrate $(200 \mathrm{ml})$ was administered at a dose of $1 \mathrm{ml} / 100 \mathrm{~g}$ body wt. and the respective control group received equivalent amounts of distilled water.

Centella asiatica Urb. (Family: Umbelliferae, S: Gotukola, T: Vallari):

Leaves of $C$. asiatica together with the stems $(600 \mathrm{~g})$ were boiled with $1500 \mathrm{ml}$ of water for 2 hours, liquidized and the squeezed through muslin cloth and filtered. The filtrate $(850 \mathrm{ml})$ was administered at a dose of $1 \mathrm{ml} / 1000 \mathrm{~g}$ body wt. and the respective control group received equivalent amounts of distilled water.

Allium sativum Linn. (Family: Amaryllidaceae, S: Sudu-lunu, T: Vellavengayam):

Cloves of garlic ( $400 \mathrm{~g}$ in weight.after removing skin) were liquidized after adding $250 \mathrm{ml}$ of water and then squeezed through muslin cloth and filtered. The filtrate (400 $\mathrm{ml}$ ) was administered at a dose of $1 \mathrm{ml} / 100 \mathrm{~g}$ body wt. and the respeciive control group received an equivalent amour

\section{Results}

Weight gain per littermate, percentage weight gain of litter and milk intake per littermate in different experimental groups are shown in Tables 1 and 2. Weight gain per littermate, percentage weight gain of litter or milk intake per littermate were not significantly different following administration of any of the plant extracts when compared with their respective control groups. However, i.m. administration of chlopromazine twice a day led to a significant increase in weight gain per littermate $(\mathrm{p}<0.05)$ and percentage increase in weight $(\mathrm{p}<0.01)$ though i.m. administration of chlopromazine once a day failed to do so.

\section{Discussion}

The animal model used appeared to be suitable to evaluate possible galactagoguic activity as administration of chlopromazine twice a day led to a significant increase in both the weight gain per littermate and percentage weight gain. The inability to observe a significant increase in milk intake following administration of chlopromazine twice a day perhaps could have been due to the fact that milk intake per littermate was measured only once during the experiment and any loss of urine or faeces by the litter 


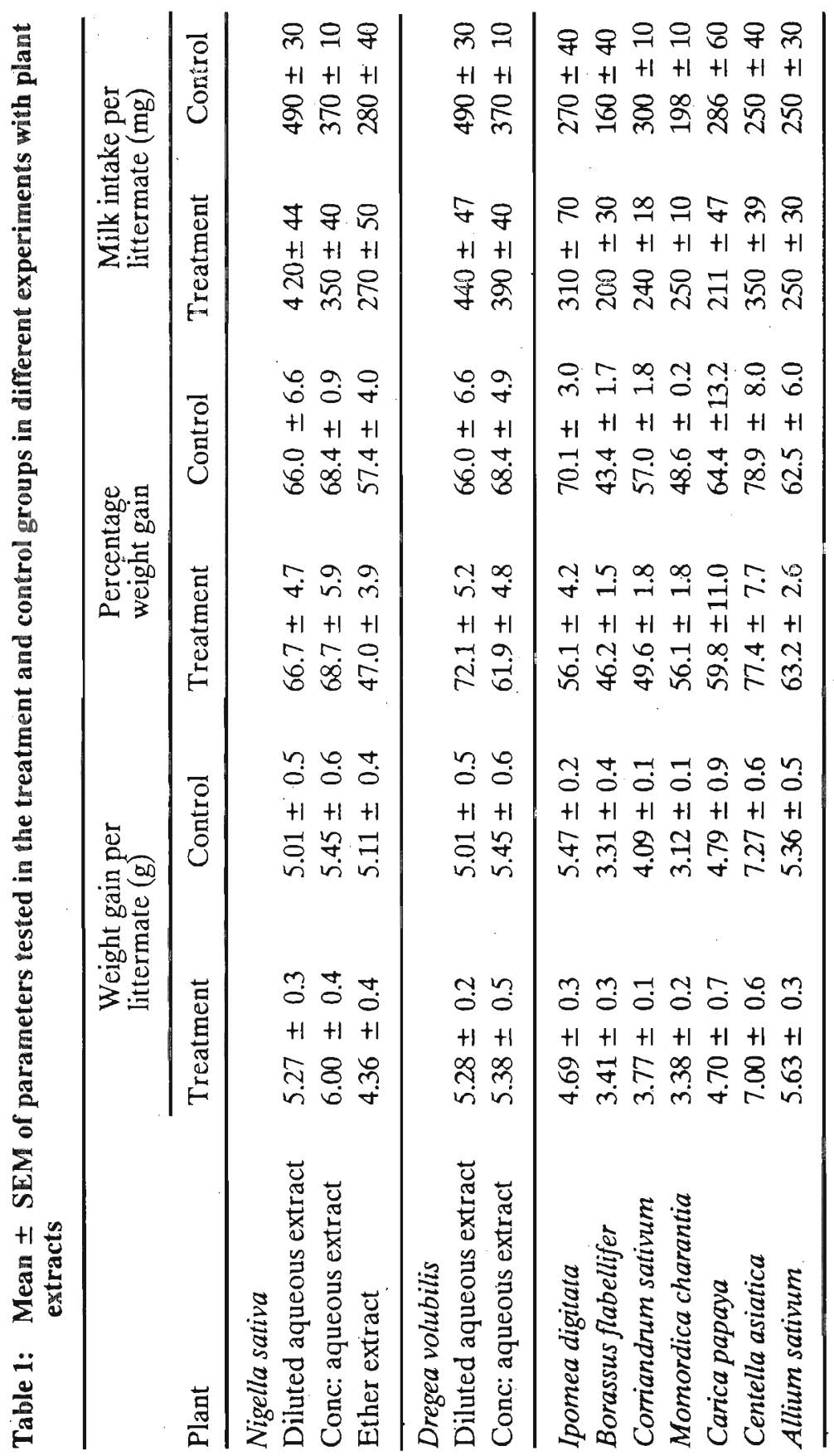




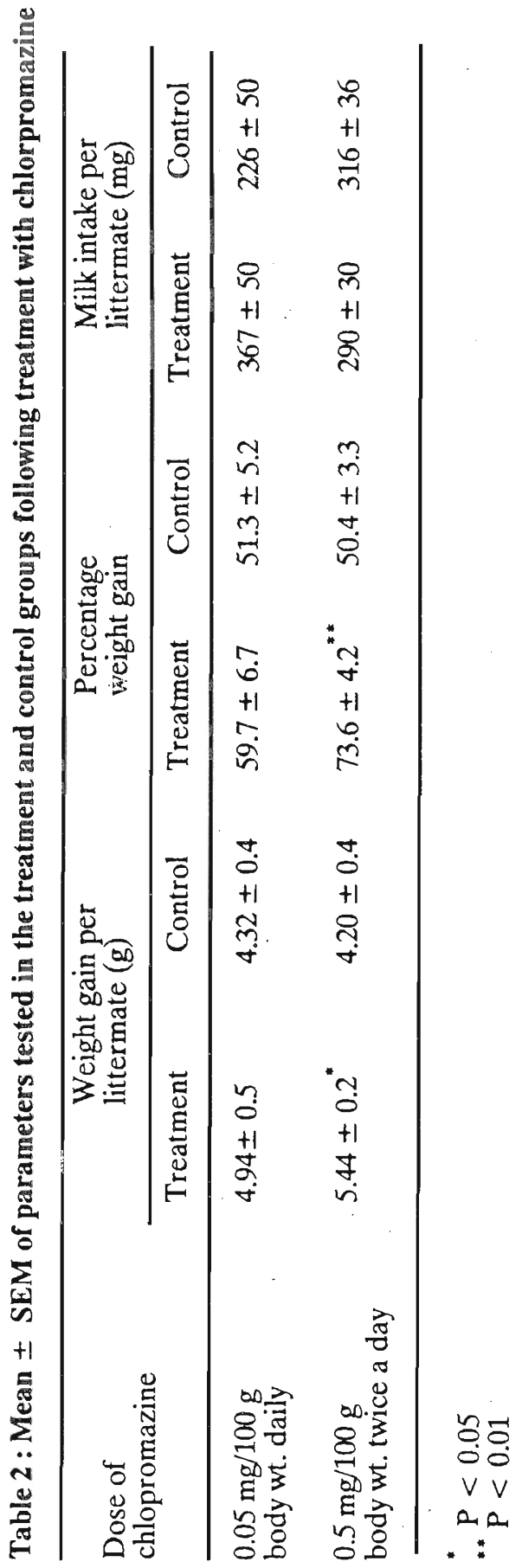


between the two weight measurements could result in a value lower than the actual milk intake.

From the plant extracts screened for possible galactagoguic activity in this study only Nigella sativa has been studied by previous investigators ${ }^{6,7}$ to our knowledge. In these studies a slight galactagoguic activity was observed when powdered $N$. sativa seeds were administered in the diet of lactating rats and weight gain of litter was used to assess the galactagoguic activity. ${ }^{6}$ Further studies by same investigators ${ }^{7}$ where ether extract of $N$. sativa was administered in the diet of lactating rats showed that the galactagoguic activity was present in the lipid fraction of $N$. sativa seeds. However when a plant extract is administered in the diet the amount ingested would be highly variable and cannot be exactly quantified. In the present study diluted aqueous, concentrated aqueous and ether extracts of $N$. sativa were administered via oral feeding tubes thus ensuring ingestion of a known quantity of the extract. Yei these studies failed to show any galactagoguic activity as assessed by the weight gain of littermate, percentage weight gain of litter or milk intake per littermate. The reason for the discrepancies between previously reported and present studies is not clear.

Only two of the previous studies reported by other investigators have used lactating rats as the animal model, oral route for administering the plant extract and litter weight or milk yield as the indicator of galactagoguic activity. An increase in the litter weight was observed following oral administration of stigmasterol and the ethir fraction of $L$. reticulata ${ }^{9}$ and an increase in the milk yield was observed following oral administration of cold water extract of $A$. augusta ${ }^{2}$ to lactating rats. Agrawala and co-workers ${ }^{6,7}$ also used administration of plant extracts in the diet of lactating rats to evaluate the galactagoguic activity of $N$. sativa and the disadvantages of administering plant material in the diet has already been mentioned.

Two other studies have measured milk yield following administration of the plart material in the diet of livestock. An increase in milk yield was observed following administration" of roots of $A$. racemosus in the diet of buffaloes ${ }^{4}$ and following administration of dried powder of $L$. reticulata in the diet of cows, buffaloes, sheep and goat. ${ }^{5}$ However, in one of these studies, a probability level less than 0.1 has been interpreted as statistically signif: cant.

Some of the previous investigators have also used changes in mammary weight or histology as indicators of galactagoguic activity. Sabnis and co-workers ${ }^{3}$ observed an increase in mammary weigint and secretory changes in mammary histology following intramuscular administration of crude alcoholic extract of $A$. racemosus to oestrogen primed rats. Secretory changes in mammary histology were also observed followirg oral administration of cold water extract of $A$. augusta to oestrogen primed rats ${ }^{2}$ and stigmasterol and ether fraction of $L$. reticulata to lactating rats. ${ }^{9}$ 
None of the plant extracts tested in the present study proved to be effective galactagogues in rats. All the plant extracts were administered in doses adequate to concentrate active principles, if there were any. When the doses used were extrapolated to humans a woman will have to eat at least $250 \mathrm{~g}$ of the concerned plant material daily to obtain the comparable dose of the extract given to a rat mother. Thus lack of galactagoguic activity in the plant extracts tested so far is unlikely to be due to inadequate dosage. All plant extracts were administered orally and the possibility of any active principles being destroyed in the digestive tract could not be excluded. However, the plants studied in the present study are recommended either as decoctions in traditional medicine or to be taken internally according to folklore and thus would be ineffective in the way it is prescribed if the active principles were to be destroyed in the gastrointestinal tract. Nevertheless, the report that plants such as L. reticulata when administered in the diet significantly increase milk yield in livestock, warrants further investigation.

Insolubility of possible active principles in water where only aqueous extracts were used and possible heat labile nature of active principles where boiling water extracts were used may also account for lack of galactagoguic activity in plants tested in the present study. Further the possibility of active principle occurring in a bound form from which it is released during extraction with organic solvents rendering it inactive during preparation may also underlie the negative findings seen in the present study. Some plants are known to show a seasonal variation in the concentrations of active principles. In this study plant material was not collected thorughout the year. Hence we cannot exclude the possibility of a seasonal variation of galactagoguic activity of the plants tested so far although it seems unlikely.

\section{Acknowledgements}

We thank T.R.K. Perera of the Animal House, Faculty of Medicine, Colombo for excellent technical assistance. This work was supported by NARESA Research Grant No: 88/M/3 and International Foundation for Science, Stockholm Research Grant No: F/1296-1.

\section{References}

1. Chaudhury R.R. \& Tennekoon K.H. (1983). Plants as galactagogues. In: Advances in International Maternal and Child Health (Eds. D.B. Jelliffe \& E.F.P. Jelliffe) 3: 20-26, Oxford University Press.

2. Venkitaraman S. \& Radhakrishnan N. (1975). Effect of Abroma augusta Linn. root on mammary growth and lactation. The Indian Journal of Pharmacy 37: 153-154. 
3. Sabnis P.B., Gaitonde B.B. \& Jetmalani M. (1968). Effects of alcoholic extract of Asparagus racemosus on mammary glands of rats. Indian Journal of Experimental Biology 6: 55-56.

4. Patel A.B. \& Kanitkar U.K. (1969). Asparagus racemosus Willd-form Bordi, as a galactagogue in buffaloes. The Indian Veterinary Journal 46: 718-721.

5. Anjaria J.V. \& Gupta L. (1967). Studies on lactogenic property of Leptadenia reticulata (Jivanti) and Leptaden tablets in goats, sheep, cows and buffaloes. The Indian Veterinary Journal 46: 718-721.

6. Agrawala I.P., Achar M.V.S., Boradkar R.V. \& Roy N. (1968). Galactagogue action of Cuminum cyminum and Nigella sativa. Indian Journal of Medical Research 56:841-844.

7. Agrawala L.P., Achar M.V.S. \& Tamankar B.P. (1971). Galactagogue action of Nigella sativa. The Indian Joumal of Medical Sciences 25: 535-537.

8. Tennekoon K.H., Karunanayake E.H. \& Mahindaratne M.P.D. (1987). Evaluation of the galactagoguic activity. of Asparagus falcatus. Ceylon Journal of Medical Sciences 30: 63-67.

9. Anjaria J.V., Varia M.R., Janakiraman K. \& Gulati O.D. (1975). Studies on Leptadenia reticulata: Lactogenic effects on rats. Indian Journal of Experimental Biology 13: 448-449. 